\title{
Caracterización de radiación solar directa y difusa en condiciones climáticas del Valle del Mantaro
}

\author{
Characterization of direct and diffuse solar radiation in weather conditions \\ of the Mantaro valley
}

\author{
Wuilber Clemente De la Cruz ${ }^{1}$, Alejandro Alcántara Boza ${ }^{2}$
}

Recibido: 01/07/2020 - Aprobado: 30/10/2020 - Publicado: 30/11/2020

\begin{abstract}
RESUMEN
En el Valle del Mantaro hay presencia significativa de la radiación solar, sin embargo, no están adecuadamente organizadas los datos que otorga las estaciones de la zona, es más, poseen una incertidumbre fuera de los límites permisibles por ser monitoreado con equipos obsoletos. Debido a que surgían investigaciones inherentes a las energías renovables, particularmente energía solar, había la necesidad de analizar y caracterizar los datos de radiación solar, a fin de que éstas sean los insumos para los diseños de sistemas solares, para tal efecto, se adquirió una estación actinométrica de alta precisión, en base a una selección y dimensionamiento adecuado, luego se instaló y se puso en operatividad, con este dispositivo se obtuvo 518000 datos durante el periodo julio 2018 a junio 2019; los mismos que han sido tratadas con la asistencia del software Excel. La caracterización nos muestra que la radiación directa de onda corta en promedio anual es de $175,7 \mathrm{~W} / \mathrm{m}^{2}$ y el máximo alcanzado fue de $553,3 \mathrm{~W} / \mathrm{m}^{2}$, generando una variación de $151,7 \mathrm{~W} / \mathrm{m}^{2}$ y $410,3 \mathrm{~W} / \mathrm{m}^{2}$, respectivamente. La radiación difusa de onda corta fue de $98,2 \mathrm{~W} / \mathrm{m}^{2}$ en promedio anual y alcanzó como máximo $297,3 \mathrm{~W} / \mathrm{m}^{2}$, con una desviación estándar de $52,7 \mathrm{~W} / \mathrm{m}^{2}$ y $170,1 \mathrm{~W} / \mathrm{m}^{2}$.
\end{abstract}

Palabras clave: Radiación directa; radiación difusa; caracterización; modelamiento; piranómetro; pirheliómetro; onda corta.

\begin{abstract}
The Mantaro Valley is a privileged area where there is a significant presence of solar radiation, however, the data emitted by the stations in the area are not properly organized, it is more, until 2017 that monitors this parameter was the Meteorological Station from Huayao but with obsolete instruments. Because research related to renewable energy, particularly solar energy, arose, there was a need to analyze and characterize solar radiation data, so that these are the inputs for solar system designs, for this purpose a station was acquired High precision actinometric at the National University of the Center of Peru-Huancayo. To achieve the purpose, the equipment was selected and acquired, then installed and put into operation, with this device 518,000 data was obtained during the period July 2018 to June 2019; the same ones that have been treated with the assistance of Excel software. The characterization shows us that the direct average shortwave radiation is $175.7 \mathrm{~W} / \mathrm{m}^{2}$ and the maximum reached was $553.3 \mathrm{~W} /$ $\mathrm{m}^{2}$, generating a variation of $151.7 \mathrm{~W} / \mathrm{m}^{2}$ and $410.3 \mathrm{~W} / \mathrm{m}^{2}$, respectively. The diffuse shortwave radiation was $98.2 \mathrm{~W} / \mathrm{m}^{2}$ on an annual average and reached a maximum of $297.3 \mathrm{~W} / \mathrm{m}^{2}$, with a standard deviation of $52.7 \mathrm{~W} / \mathrm{m}^{2}$ and $170.1 \mathrm{~W} / \mathrm{m}^{2}$.
\end{abstract}

Keywords: Direct radiation; diffuse radiation; characterization; modeling; pyranometer; pyrometer; short wave.

\footnotetext{
1 Estudiante del Doctorado en Ciencias Ambientales. Universidad Nacional Mayor de San Marcos, Facultad de Ingeniería Geológica, Minera, Metalúrgica y Geográfica. Lima, Perú. Ingeniero. Maestría en Geología con mención en Minas y Recursos Energéticos.

Autor para correspondencia: wuilber.clemente@unmsm.edu.pe ORCID: https://orcid.org/0000-0002-2634-7737

2 Docente Permanente de la Universidad Nacional Mayor de San Marcos, Facultad de Ingeniería Geológica, Minera, Metalúrgica y Geográfica. Lima, Perú. Magister. E-mail: falcantarab@unmsm.edu.pe ORCID: https://orcid.org/0000-0001-9127-4450
} 


\section{INTRODUCCIÓN}

El Valle del Mantaro, se extiende a lo largo de las provincias; Huancayo, Concepción, Chupaca y Jauja; departamento de Junín, a una altitud de 3300 m.s.n.m.,

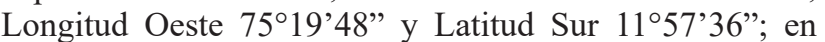
ello, existen gradientes superficiales que hacen variar la altitud, latitud y longitud, esto hace que los parámetros actinométricos también varíen, es decir, la radiación solar de onda corta tiene un comportamiento divergente en cada zona. Para determinar con precisión y exactitud estas variaciones, específicamente de la radiación solar directa y difusa, se realizaron las mediciones con instrumentos calibradas en diferentes puntos del Valle, y los resultados se discriminaron mostrando el grado de incertidumbre del mensurando. Posteriormente, se realizó la caracterización a fin de establecer y definir las irradiancias directa y difusa en función a la altitud y así establecer conclusiones significativas del comportamiento de la radiación a lo largo del tiempo. Las energías renovables en el contexto actual tiene significancia y todos los países del mundo están impulsando su difusión y desarrollo, es más están denominándolo como energía del futuro por su compatibilidad con el medio ambiente, tal es así, el papel de la energía renovable como alternativa al poder basado en fósiles está creciendo en el mundo en desarrollo (Zawilska \& Brooks, 2011). En particular, el que tiene más auge es la energía solar, en consecuencia, merece una atención minuciosa y meticulosa en su análisis y evaluación. Además la utilización de la energía solar es cada vez más importante para satisfacer las necesidades futuras de energía y limitar las emisiones de CO2 a la atmósfera (Gelsor et al., 2018). La búsqueda necesaria para un futuro global más sostenible significa usar fuentes de energía renovables para generar electricidad libre de contaminantes, es así, las plantas CSP (energía solar concentrada) y fotovoltaica (fotovoltaica) son los sistemas más demandados para la producción de electricidad utilizando la radiación solar como fuente de energía (Alonso-Montesinos \& Batlles, 2015). El diseño y la evaluación del rendimiento del sistema solar fotovoltaico y térmico requiere el conocimiento de la radiación solar global (Manju \& Sandeep, 2019). La radiación solar y la temperatura ambiente son los recursos básicos para el despliegue a gran escala de tecnologías de energía solar (Dhimish \& Mather, 2019). La explotación de la energía solar en sistemas solares térmicos y fotovoltaicos requiere el análisis de los datos de radiación solar y los modelos utilizados para evaluar el rendimiento de los sistemas (Jamil \& Akhtar, 2017). Para caracterizar la radiación solar, se genera y analiza en una estación actinométrica, un conjunto de datos de la radiación solar horizontal global diaria resultante de las series de tiempo de observaciones in situ a largo plazo (Journée et al., 2012). La caracterización del recurso solar de un sitio es esencial para las diferentes fases de los proyectos de energía solar y la profundidad requerida de la evaluación aumenta a medida que avanza el proyecto, incluidas las estimaciones a largo plazo que solo se pueden obtener. a través de un análisis estadístico de una base de datos continua y a largo plazo de mediciones de radiación solar (Lillo-bravo \& Ramı, 2016). Todos los componentes de radiación solar presentan un ciclo diurno bien definido con un máximo al mediodía, las estimaciones de la radiación solar directa y global indican un gran potencial disponible para la energía solar en la superficie, especialmente en verano (Marques Filho et al., 2016).

En consecuencia, su medición o evaluación requieren las escalas temporales intrínsecas de la variabilidad de la radiación solar de superficie y se abordan mediante la descomposición del modo empírico, es más, se analizan las mediciones de alta calidad de la radiación solar que incide sobre un plano horizontal a nivel del suelo, desde diferentes estaciones terrestres (Bengulescu et al., 2016). La irradiación horizontal global, la irradiancia horizontal difusa, la irradiancia normal directa, la temperatura de la superficie, la velocidad del viento y la dirección del viento y la humedad relativa deben ser medidos y analizados durante un año completo como mínimo en cada estación, los datos se filtran para garantizar su consistencia y calidad (Parinacota et al., 2017). Los sesgos porcentuales promedio de las mediciones en estaciones deben rodear a $5 \%$ para GHI y $10 \%$ para DNI (Sengupta et al., 2019) Para la mayoría de las aplicaciones de energía solar, el primer paso es una información precisa de la cantidad de radiación solar recibida por una superficie horizontal, para ello es necesario que los instrumentos estén calibrados y así poder estimar la radiación solar global diaria (DGSR) en una superficie horizontal utilizando datos a largo plazo (Gouda et al., 2019). Para evaluar el rendimiento de los sistemas solares térmicos o fotovoltaicos, y en el caso de pronósticos climáticos a largo y corto plazo, la radiación solar debe descomponerse en sus fracciones difusas y de haz para predecir la cantidad correcta de energía solar involucrada en un contexto particular (Scarpa et al., 2018). La cuantificación de la radiación solar incidente en una superficie es una tarea compleja que requiere el conocimiento de las características geométricas, geográficas, astronómicas, físicas y meteorológicas de la ubicación (GutiérrezTrashorras et al., 2018). El modelo es el que demuestra el comportamiento generalizado para evaluar la radiación solar global en cualquier lugar de la región (Anis, Shahrukh et al., 2019), es más, puede usarse con éxito para estimar la radiación solar durante todas las estaciones del año para la posición estudiada y para el día considerado, utilizando como entrada la altitud, la longitud y la latitud (El Mghouchi et al., 2014). En el siglo XXI, los modelos y mediciones actuales de radiación solar son bastante comparables, con incertidumbres de medición absolutas del orden de 25-100 $\mathrm{W} / \mathrm{m} 2$ en datos medidos hemisféricos, $\mathrm{y} \pm 25 \mathrm{~W} / \mathrm{m} 2$ en datos medidos por haz directo (Khalil, 2008).

\section{METODOS}

Para la obtención de datos de la radiación solar directa y difusa, en primera instancia se seleccionaron los equipos de medición, luego se definió el lugar de instalación, tal es así, la estación actinométrica se instaló en la azotea del edificio central de la Universidad Nacional del Centro del Perú, Huancayo; es decir, la estación está ubicado en una superficie plana horizontal, a una altura de $34 \mathrm{~m}$ respecto a la superficie del lugar, específicamente a 3314 m.s.n.m. a una latitud de $12^{\circ} 3^{\prime} 54.5^{\prime \prime} \mathrm{S}$ y longitud Oeste $75^{\circ} 12^{\prime} 17.5^{\prime \prime}$ O. El lugar se encuentra libre de sombras y están instalados los siguientes equipos: Un Piranómetro CMP 21 para monitorear la radiación global y un Piranómetro CMP 21 para radiación difusa; ambos tienen un rango de monitoreo hasta $4000 \mathrm{~W} / \mathrm{m} 2$, clasificación a ISO 9060: 2018, El 
Pirheliómetro $\mathrm{CH} 1$ nos permite medir la radiación directa cuyo alcance es hasta $4000 \mathrm{~W} / \mathrm{m} 2$ avalado por la norma internacional ISO de primera clase; los mismos que están montados sobre un Tracker solar SOLYS 2 cuya puntería de seguimiento pasivo se halla entre $0.1^{\circ}$ y $0.2^{\circ}$ y seguimiento activo con sensor solar, además permite su interacción via Ethernet/interfaz web, que sigue al movimiento del sol. Todos ellos tienen un sistema de control, para ello se instaló un Datalogger marca CAMBELL CR 1000X, el mismo que registra datos cada minuto en promedio (es decir los datos que capta en menos de un segundo lo promedia), tal es así, su precisión de reloj en tiempo real es \pm 3 min por año (corrección GPS opcional $\pm 10 \mu \mathrm{s}$ ).

El objeto de la investigación está constituido fundamentalmente por la interacción de la radiación solar y los instrumentos de medición tales como: Piránómetro CMP21 (global) Kipp Zonen, Piranómetro CMP2 (difusa), Pirheliómetro CH1 (directa), Tracker solar y el Datalogger; todos ellos son accionados por la energía eléctrica. La radiación solar incide sobre los equipos y estos captan los fotones y lo emiten hacia el Registrador de datos en forma de radiación medidos en $\mathrm{Wh} / \mathrm{m} 2$. La medición se realizó en todo momento con una precisión y exactitud significativa.

Para la obtención de datos se cumplió con el procedimiento, tal como muestra la figura 1 y figura 2 .
A partir del Datalogger se inicia la descarga a través del software PC200 y se exporta al Excel para su respectiva organización. La tabla 1 muestra los instrumentos y los parámetros medidos

\section{RESULTADOS}

El datalogger registra datos a cada minuto, es decir, en una hora se registra 60 datos, en un día 1440, en un mes 43200 y en un año 518400 datos; a partir de este último se organiza y se hace el tratamiento con fines de representar en tablas y gráficos de manera comprimida.

\subsection{Caracterización de la radiación directa}

La medición de la radiación solar directa en $\mathrm{W} / \mathrm{m}^{2}$ se hizo en el periodo de un año a través del Pirheliómetro Kipp Zonen, se inició el primero de julio del año 2018 y se culminó el 30 de junio del 2019; en este intervalo se tuvo 518400 datos de radiación directa y la misma cantidad en radiación difusa. La organización se hizo por cada mes y dentro de éste se organizó por horas, es decir; en el eje de las abscisas ubicamos las horas y en el eje de las ordenadas ubicamos los promedios de las radiaciones incidentes directos en $\mathrm{W} / \mathrm{m}^{2}$, de los 30 días del mes, tal como se aprecian en la figura 3.

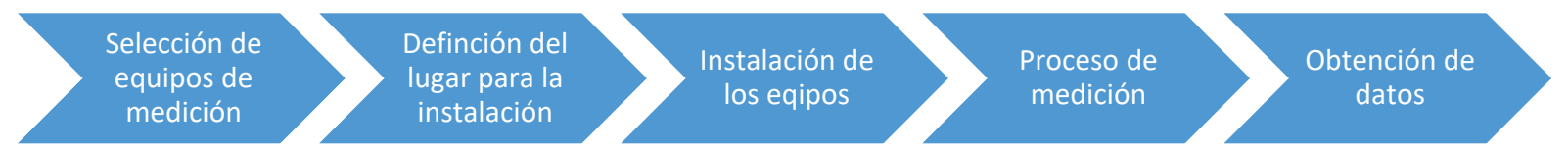

Figura 1. Procedimiento para la obtención de datos.

Fuente: Elaboración propia

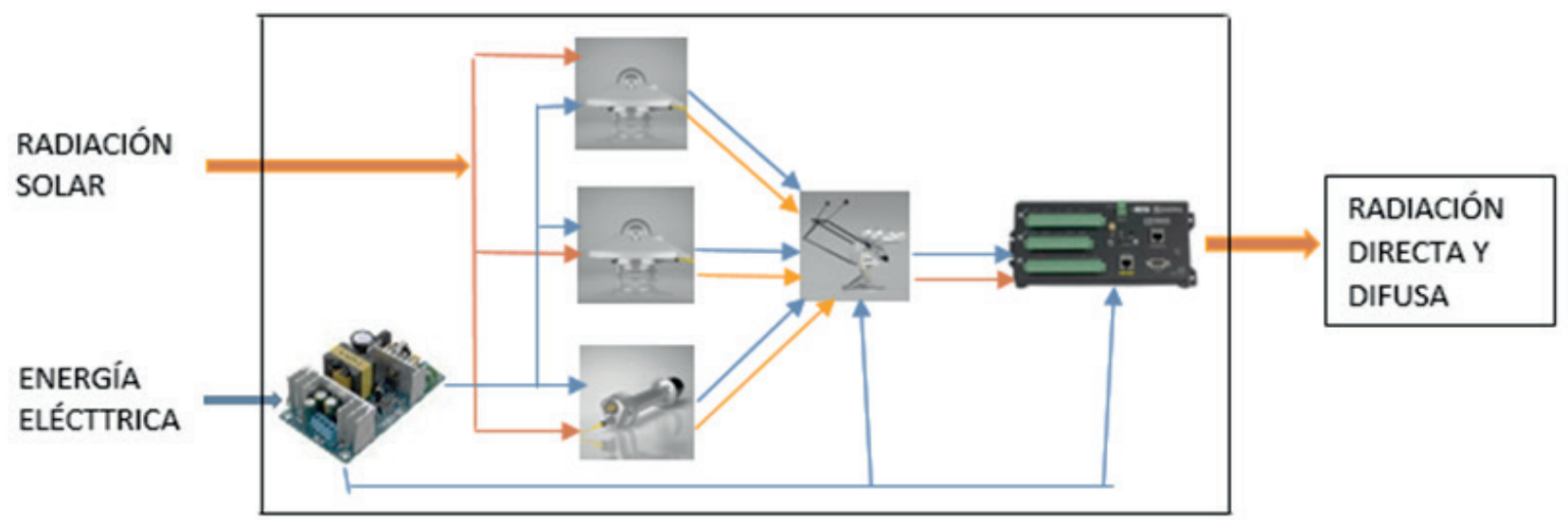

Figura 2. Objeto de estudio de la investigación.

Fuente: Elaboración propia

Tabla 1. Instrumentos y los parámetros medidos

\begin{tabular}{lllll}
\multicolumn{1}{c}{ Nomenclatura } & \multicolumn{1}{c}{ Descripción } & \multicolumn{1}{c}{ Instrumento } & Modelo & Marca \\
\hline CHP1_SlrW_Avg & Radiación solar directa incidente & Pirheliómetro & CHP1 & Kipp Zonen \\
CMP21_SlrW_2_Avg & Radiación solar difusa de onda corta & Piranómetro & CMP21 & Kipp Zonen \\
\hline
\end{tabular}

Fuente: Elaboración propia 


\subsection{Caracterización de la radiación difusa}

La medición de la radiación solar difusa de onda corta se realizó a través de un Piranómetro Kipp Zonen CMP
21_SlrW_2_Avg, en el mismo intervalo de tiempo que la radiación directa, en $\mathrm{W} / \mathrm{m}^{2}$, los mismos que se muestran en la figura 4.
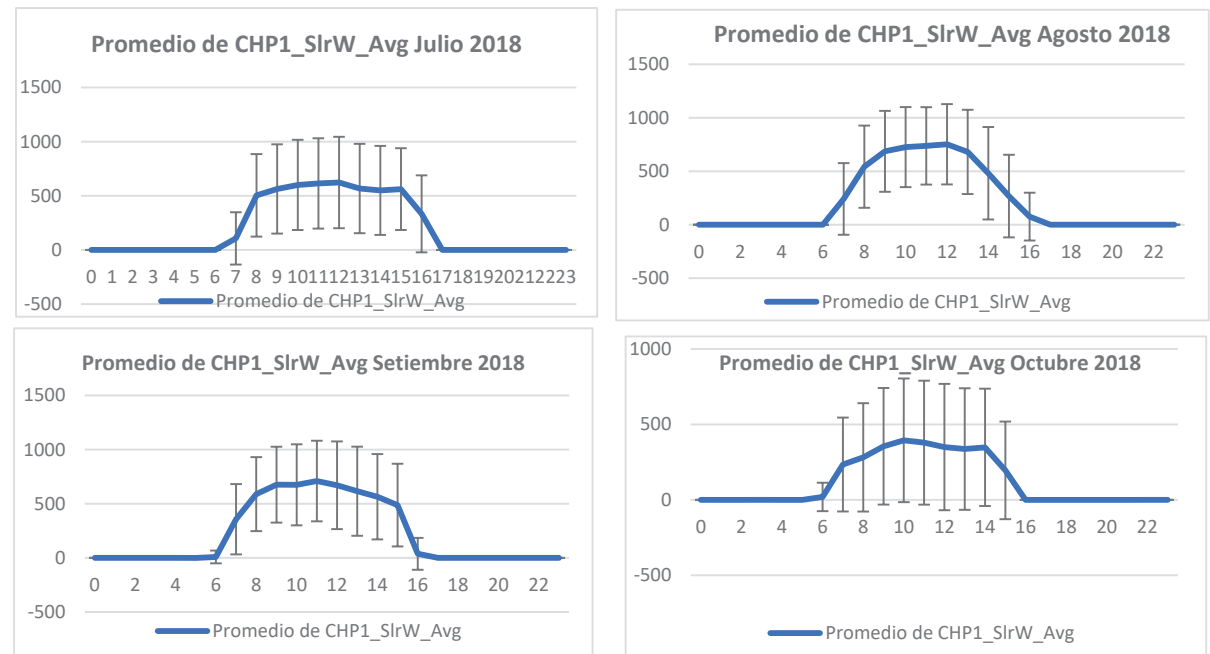

Promedio de CHP1_SIrW_Avg Nov. 2018

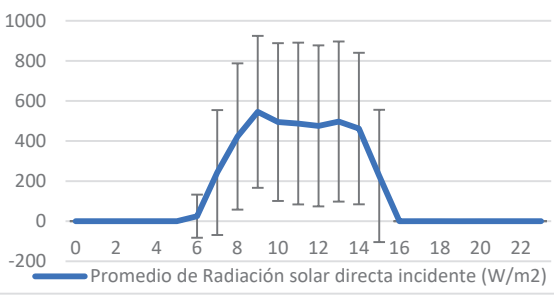
1000

Promedio de CHP1_SIrW_Avg enero 2019

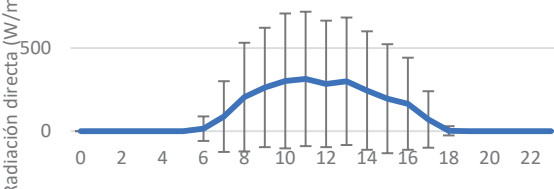

Promedio de RHGrááión solar directa.

Promedio de CHP1_SIrW_Avg marzo

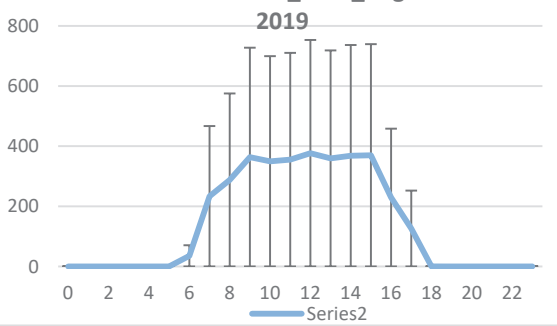

Promedio de Radiación directa Mayo 2019 $(\mathrm{W} / \mathrm{m} 2)$
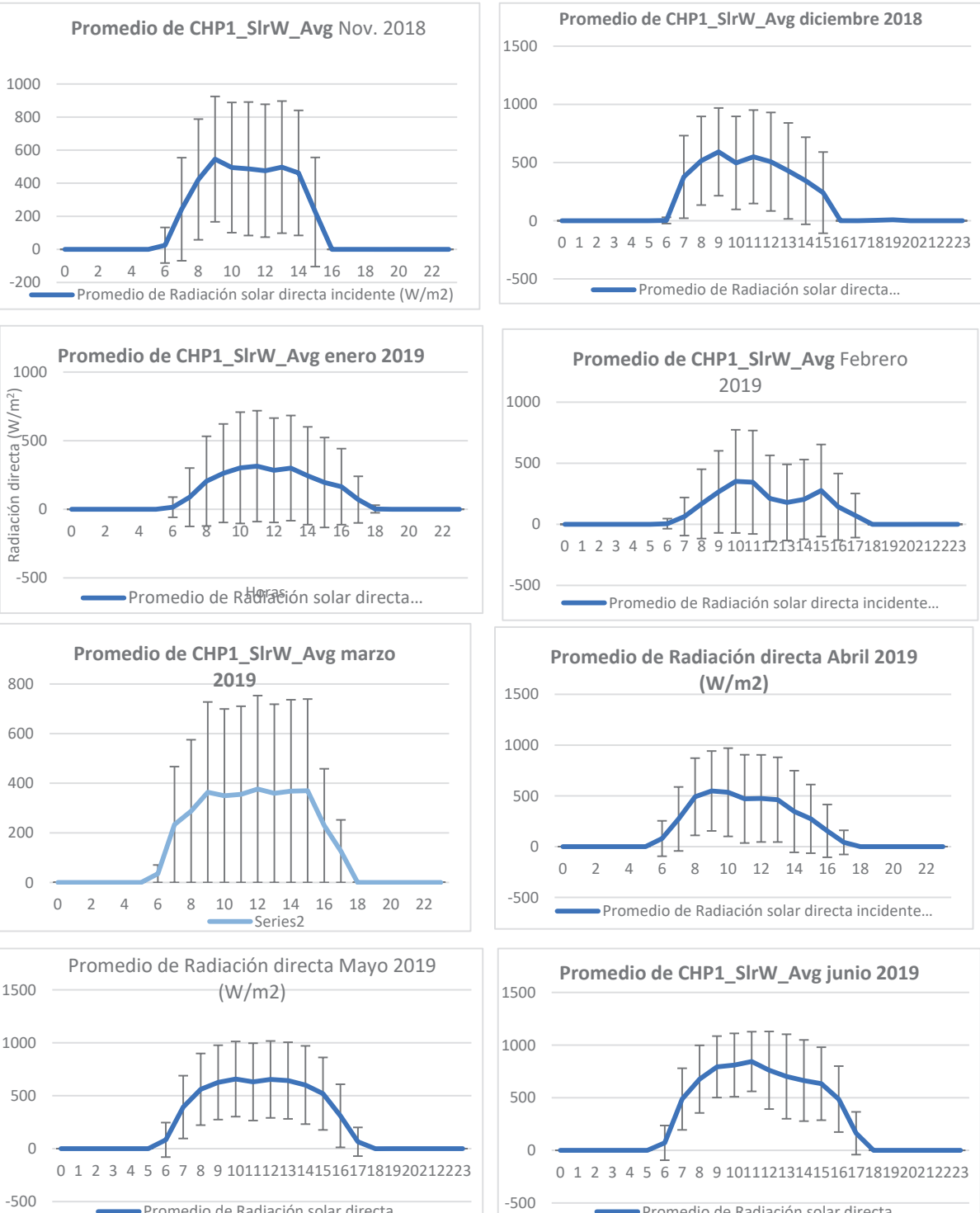

$-500 \longrightarrow$ Promedio de Radiación solar directa...

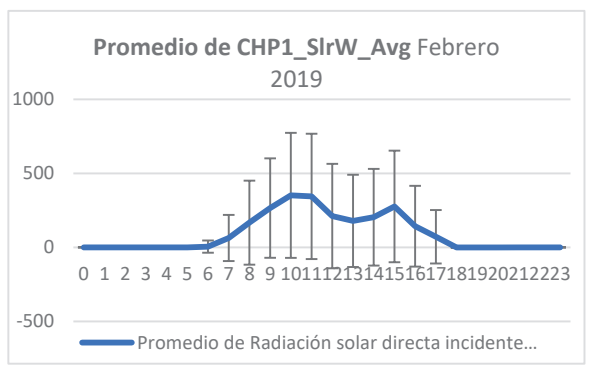

Promedio de Radiación directa Abril 2019 (W/m2)

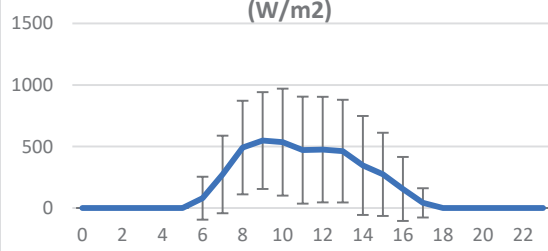

$-500$

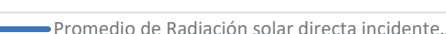

Promedio de CHP1_SIrW_Avg junio 2019

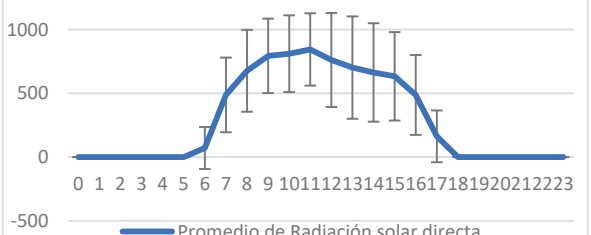

Figura 3. Radiación solar directa en W/m² de los doce meses (junio 2018-julio 2019). 


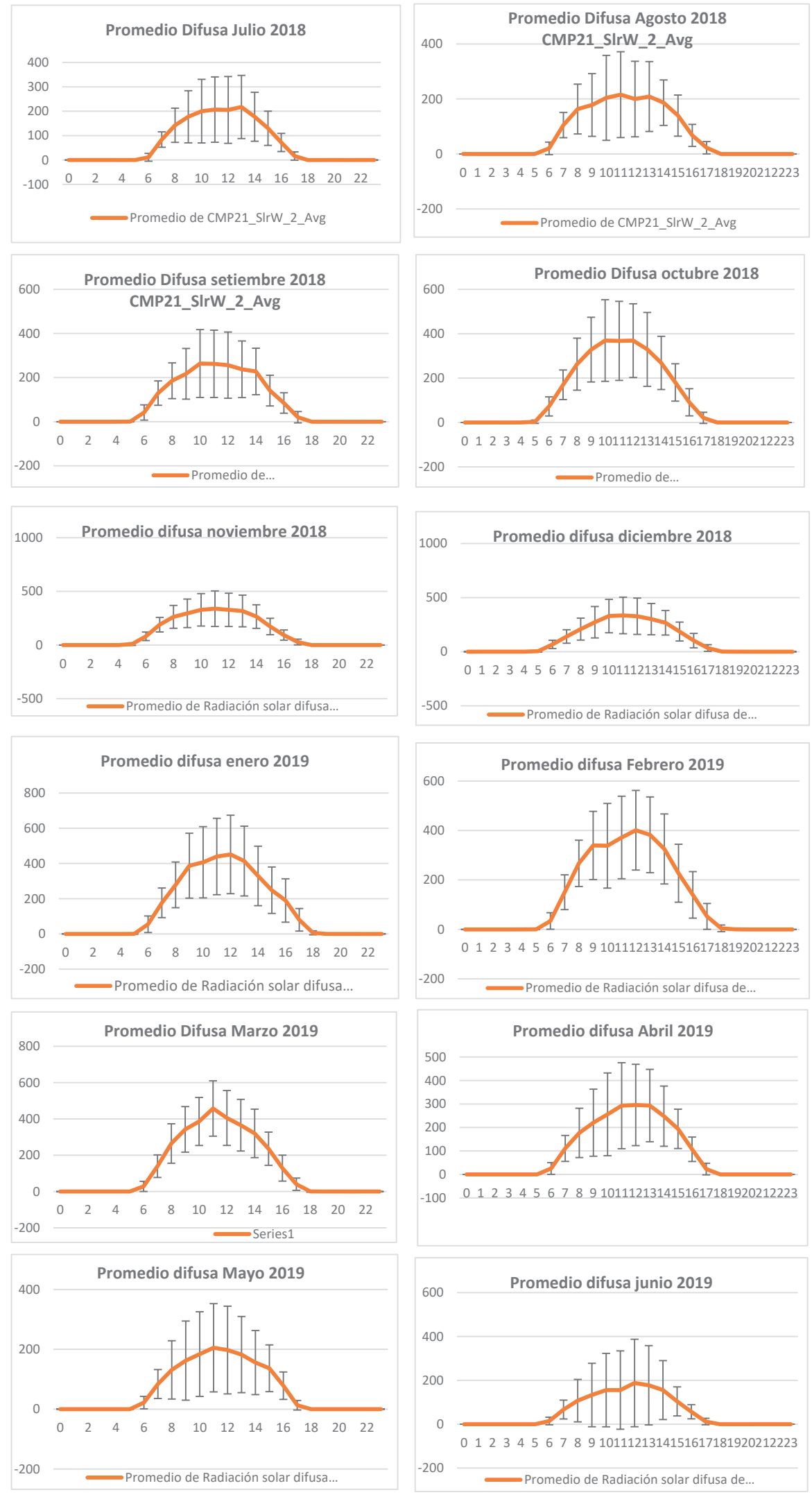

Figura 4. Radiación difusa promedio de onda corta y desviación estándar del periodo julio 2018 al junio del 2019

El tratamiento estadístico de los datos se realizó con la asistencia del software Excel, en particular de las tablas dinámicas y sus barras de error; los mismos permitieron determinar la variación de la radiación solar directa y difusa. Además, se muestra la variación de la radiación a lo largo del mes y como muestra tomamos al mes de junio del 2019, ver figura 5. 
Todos los meses del año posee similar configuración del gráfico, lógicamente varía en la cuantificación

El comportamiento de la radiación directa y difusa tiene cierta similitud, y tienen una configuración polinómica de sexto orden, y está representado por:

$$
\mathrm{y}=-0.0011 \mathrm{x}^{6}+0.0845 \mathrm{x}^{5}-2.2723 \mathrm{x}^{4}+26.387 \mathrm{x}^{3}-
$$
$121.89 x^{2}+201.72 x-90.441$

Con una correlación significativa de $\mathrm{R}=0,9562$

En resumen, la caracterización consiste en presentar los valores máximos, mínimos, y media, tanto de la radiación directa y su Desviación Estándar o variación, todo ello podemos apreciar en la tabla 2.

\section{DISCUSIÓN}

Según Anis, Shahrukh et al. (2019), presentan en su artículo los diferentes modelos de estimación de radiación solar en India, como energía, es decir en $\mathrm{MJ} /\left(\mathrm{m}^{2} \mathrm{xdía}\right)$, que bordea a 20. En nuestro estudio, convirtiendo a energía tenemos como mínimo $8,2 \mathrm{MJ} /\left(\mathrm{m}^{2} \mathrm{x}\right.$ día $)$ y como máximo 72,8 $\mathrm{MJ} / /\left(\mathrm{m}^{2} \mathrm{xdía}\right)$, es decir, el Valle del Mantaro tiene una potencialidad en el recurso solar. Asimismo, en Pakistán la radiación bordea de $7,5 \mathrm{~kW} / \mathrm{m}^{2}$ a $8 \mathrm{~kW} / \mathrm{m}^{2}$ (Tahir \& Asim, 2018), en nuestro consolidado de resultados la radiación varía de $0,95 \mathrm{~kW} / \mathrm{m}^{2} \mathrm{x}$ día hasta $8,4 \mathrm{~kW} / \mathrm{m}^{2} \mathrm{x}$ día, es decir la presencia del sol es muy variable.

Según Gouda et al. (2019), hace notar la presencia de irradiancia promedio anual diario de $3,6 \mathrm{~h}$ a 8 horas y en este intervalo la irradiancia oscila entre $10,4 \mathrm{MJ} / \mathrm{m}^{2} \mathrm{x}$ día a

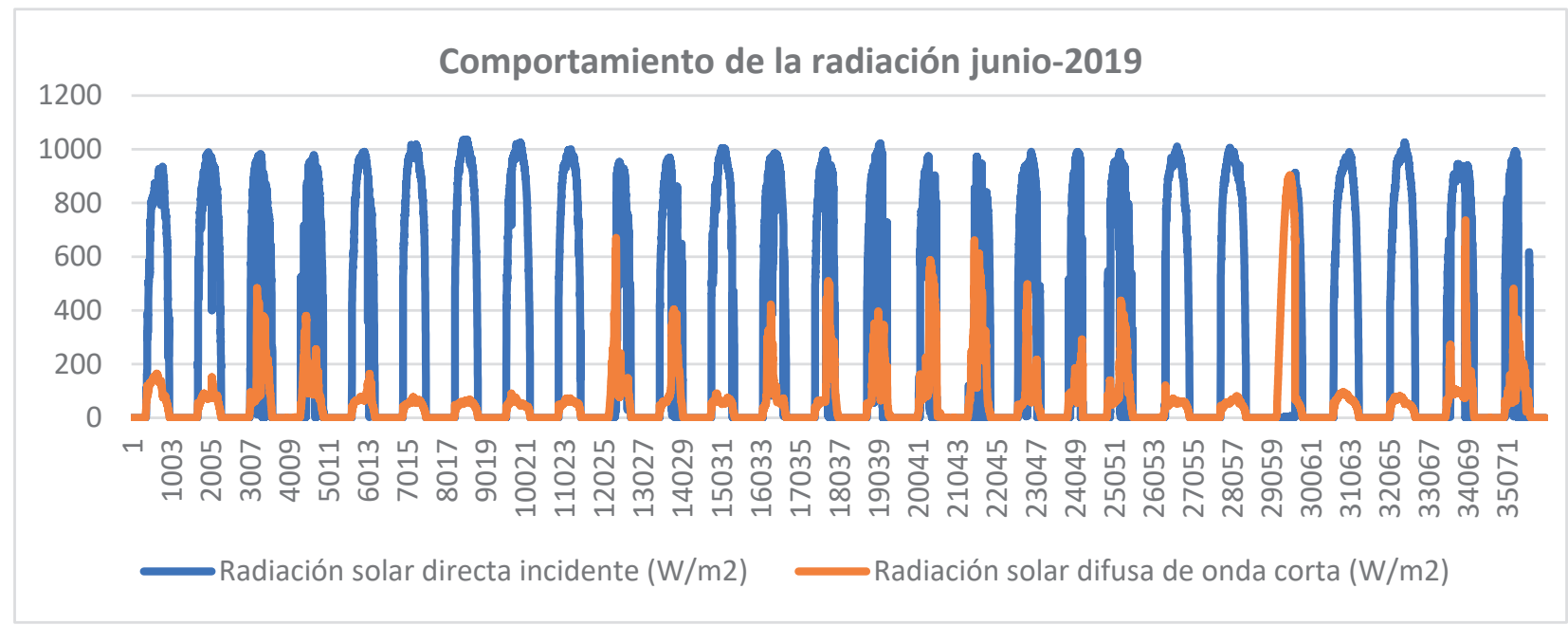

Figura 5. Variación de la radiación solar a lo largo del mes de junio 2019.

Tabla 2. Resumen de la caracterización de la radiación directa y difusa de onda corta

\begin{tabular}{cccccccccccccc}
\hline \multirow{2}{*}{ Mes-Año } & \multicolumn{3}{c}{ Rad-Directa } & \multicolumn{3}{c}{ Desvestp. Directa. } & \multicolumn{3}{c}{ Rad-Difusa } & \multicolumn{3}{c}{ Desvestp. Difusa } \\
& Min & Media & Max & Min & Media & Max & Min & Media & Max & Min & Media & Max \\
\hline Jul-18 & 0 & 209.184 & 622.432 & 0 & 160.271 & 421.949 & 0 & 40.875 & 137.020 & 0 & 40.875 & 137.020 \\
Ago-18 & 0 & 216.287 & 752.3173 & 0 & 151.9194 & 432.2944 & 0 & 44.533 & 156.119 & 0 & 44.533 & 156.119 \\
Set-18 & 0 & 224.5742 & 709.6602 & 0 & 148.4606 & 411.6277 & 0 & 46.61503 & 153.7498 & 0 & 46.61503 & 153.7498 \\
Oct-18 & 0 & 120.6415 & 394.6842 & 0 & 146.1378 & 418.9347 & 0 & 56.91463 & 183.7819 & 0 & 56.91463 & 183.7819 \\
Nov-18 & 0 & 161.6211 & 545.6513 & 0 & 144.5734 & 403.6549 & 0 & 51.67188 & 165.6989 & 0 & 51.67188 & 165.6989 \\
Dic-18 & 0 & 169.2985 & 591.786 & 0 & 145.8299 & 423.3072 & 0 & 53.90067 & 168.9247 & 0 & 53.90067 & 168.9247 \\
Ene-19 & 0 & 102.0403 & 314.2274 & 0 & 154.3509 & 405.6909 & 0 & 74.49678 & 222.7601 & 0 & 74.49678 & 222.7601 \\
Feb-19 & 0 & 95.17861 & 351.5772 & 0 & 145.3122 & 423.2366 & 0 & 58.6369 & 171.1973 & 0 & 58.6369 & 171.1973 \\
Mar-19 & 0 & 102.0317 & 308.6622 & 0 & 143.9261 & 376.6168 & 0 & 51.56929 & 152.4938 & 0 & 51.56929 & 152.4938 \\
Abr-19 & 0 & 172.9235 & 548.1917 & 0 & 170.7701 & 434.7544 & 0 & 54.3304 & 182.9808 & 0 & 54.3304 & 182.9808 \\
May-19 & 0 & 239.2533 & 657.3719 & 0 & 155.8378 & 369.5833 & 0 & 46.24803 & 147.5399 & 0 & 46.24803 & 147.5399 \\
Jun-19 & 0 & 295.3094 & 843.5467 & 0 & 153.0453 & 401.355 & 0 & 53.19015 & 199.4556 & 0 & 53.19015 & 199.4556 \\
\hline
\end{tabular}

Fuente: Elaboración propia 
$17 \mathrm{MJ} / \mathrm{m}^{2} \mathrm{x}$ día, y nuestro estudio demuestra que podemos llegar hasta máximo $72,8 \mathrm{MJ} / /\left(\mathrm{m}^{2} \mathrm{x}\right.$ día $)$.

Otros estudios muestran resultados divergentes, debido a su particularidad, tal es así, en India tiene una abundante disponibilidad de energía solar durante todo el año con un promedio anual de radiación solar global de más de 5,25 kWh / m2-día 3 con una luz solar del orden de 2776 horas anuales (Jamil \& Akhtar, 2017). Además, esta energía puede alcanzar un máximo de $7,5 \mathrm{kWhr} / \mathrm{m}^{2} x$ día durante el período de verano (abril-mayo) en partes del norte de la India (Jamil \& Akhtar, 2017). En caso nuestro, el análisis se hizo a la radiación directa y difusa y no del global, sin embargo, podemos estimar con el intervalo del tiempo de radiación por día que es 8 horas y la suma de radiación difusa y directa, por tanto, la radiación global

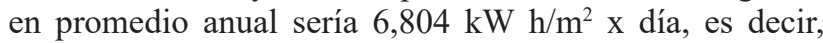
significativamente mayor que la radiación de la India. Sin embargo, existe países que tienen alta radiación, tal como se muestra en el estudio Evaluación exploratoria de la radiación solar y la temperatura ambiente en veinte lugares distribuidos en el Reino Unido, en el que la radiación

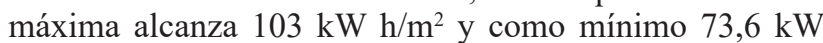
$\mathrm{h} / \mathrm{m}^{2}$, esto realmente es superior a la nuestra (Dhimish \& Mather, 2019).

El estudio "Caracterización de la variabilidad temporal en medidas de radiación solar de superficie y su dependencia del clima", presenta la variación de la radiación solar directa, el mismo que oscila entre $175 \mathrm{~W} /$ $\mathrm{m}^{2}$ y $186 \mathrm{~W} / \mathrm{m}^{2}$ en el periodo de 2002 a 2008 (Bengulescu et al., 2016), en nuestro estudio esta radiación es similar, en promedio anual es $176 \mathrm{~W} / \mathrm{m}^{2}$, adicionalmente nosotros hicimos el análisis de la variación de las radiaciones directa y difusa, en comparación a otros estudios mencionados.

\section{CONCLUSIONES}

La caracterización de la radiación solar directa, permitió establecer que el intervalo donde hay presencia de los rayos solares es de unos $8 \mathrm{~h}$ a $11 \mathrm{~h}$ al día, esto hace que la radiación directa máxima puede alcanzar a $9,3 \mathrm{kWh} / \mathrm{m}^{2}$ por día en el mes de junio y $3,5 \mathrm{kWh} / \mathrm{m}^{2}$ en el mes de febrero.

La radiación directa de onda corta en promedio anual arroja $175,7 \mathrm{~W} / \mathrm{m}^{2}$, y como máximo es de $553,3 \mathrm{~W} / \mathrm{m}^{2}$ y su variación o desviación estándar oscila entre 151.703 410.250, respectivamente; este último indica la presencia de nubes y partículas en el espacio, en algunos casos la variación es mayor a la radiación directa, es decir, en mayor porcentaje de los días hubo presencia de nubes.

La radiación difusa de onda corta también es significativo, tal es así, el promedio es $98,2 \mathrm{~W} / \mathrm{m}^{2}$ y el máximo es $297,3 \mathrm{~W} / \mathrm{m}^{2}$ y su variación se halla entre 52,7 y 170,1 , respectivamente, esto corrobora la existencia de las nubes en el espacio. La radiación global es la suma de estos dos tipos de radiaciones y son éstas las que se aprovechan para diseñar dispositivos o sistemas solares de diverso uso.

Entre enero y marzo tiene mayor incidencia la radiación difusa, y por su naturaleza, el Valle del Mantaro en esas épocas es mayormente nublado. Generalmente en los meses de junio, julio, agosto y setiembre, la radiación directa es mayor que la difusa, debido a poca presencia de la nube, sin embargo, existe variación considerable, esto debido a la presencia de partículas e impurezas en el espacio.

\section{AGRADECIMIENTOS}

Este trabajo fue financiado por el CONCYTECFONDECYT en el marco de la convocatoria E041-01 [número de contrato 76-2018-FONDECYT-BM-IADTMU].

\section{REFERENCIAS}

Alonso-Montesinos, J., \& Batlles, F. J. (2015). The use of a sky camera for solar radiation estimation based on digital image processing. Energy, 90, 377-386. https://doi.org/10.1016/j. energy.2015.07.028

Anis, Shahrukh, M., Jamil, B., Azeem Ansari, M., \& Bellos, E. (2019). Generalized models for estimation of global solar radiation based on sunshine duration and detailed comparison with the existing: A case study for India. Sustainable Energy Technologies and Assessments, 31, 179-198. https://doi.org/10.1016/j.seta.2018.12.009

Bengulescu, M., Blanc, P., \& Wald, L. (2016). Characterizing Temporal Variability in Measurements of Surface Solar Radiation and its Dependence on Climate. Energy Procedia, 97, 164-171. https://doi.org/10.1016/j.egypro.2016.10.045

Dhimish, M., \& Mather, P. (2019). Exploratory evaluation of solar radiation and ambient temperature in twenty locations distributed in United Kingdom. Urban Climate, 27, 179192. https://doi.org/10.1016/j.uclim.2018.12.001

El Mghouchi, Y., El Bouardi, A., Choulli, Z., \& Ajzoul, T. (2014). New model to estimate and evaluate the solar radiation. International Journal of Sustainable Built Environment, 3(2), 225-234. https://doi.org/10.1016/j.ijsbe.2014.11.001

Gelsor, N., Gelsor, N., Wangmo, T., Chen, Y. C., Frette, Ø., Stamnes, J. J., \& Hamre, B. (2018). Solar energy on the Tibetan Plateau: Atmospheric influences. In Solar Energy (Vol. 173, pp. 984-992). https://doi.org/10.1016/j. solener.2018.08.024

Gouda, S. G., Hussein, Z., Luo, S., \& Yuan, Q. (2019). Model selection for accurate daily global solar radiation prediction in China. Journal of Cleaner Production, 221, 132-144. https://doi.org/10.1016/j.jclepro.2019.02.211

Gutiérrez-trashorras, A. J., Villicaña-ortiz, E., Álvarez-álvarez, E., González-caballín, J. M., Xiberta-bernat, J., \& Suarezlópez, M. J. (2018). Attenuation processes of solar radiation . Application to the quanti fi cation of direct and di ff use solar irradiances on horizontal surfaces in Mexico by means of an overall atmospheric transmittance. Renewable and Sustainable Energy Reviews, 81(June 2017), 93-106. https://doi.org/10.1016/j.rser.2017.07.042

Jamil, B., \& Akhtar, N. (2017). Comparison of empirical models to estimate monthly mean diffuse solar radiation from measured data: Case study for humid-subtropical climatic region of India. In Renewable and Sustainable Energy Reviews (Vol. 77, pp. 1326-1342). https://doi.org/10.1016/j. 


\section{rser.2017.02.057}

Journée, M., Müller, R., \& Bertrand, C. (2012). Solar resource assessment in the Benelux by merging Meteosat-derived climate data and ground measurements. Solar Energy, 86(12), 3561-3574. https://doi.org/10.1016/j.solener.2012.06.023

Khalil, S. A. (2008). Parameterization models for solar radiation and solar technology applications. Energy Conversion and Management, 49(8), 2384-2391. https://doi.org/10.1016/j. enconman.2008.01.023

Lillo-bravo, I., \& Ram1, L. (2016). Solar resource assessment in Seville, Spain. Statistical characterisation of solar radiation at different time resolutions. 132, 430-441. https:// doi.org/10.1016/j.solener.2016.03.032

Manju, S., \& Sandeep, M. (2019). Prediction and performance assessment of global solar radiation in Indian cities: A comparison of satellite and surface measured data. Journal of Cleaner Production, 230, 116-128. https://doi. org/10.1016/j.jclepro.2019.05.108

Marques Filho, E. P., Oliveira, A. P., Vita, W. A., Mesquita, F. L. L., Codato, G., Escobedo, J. F., Cassol, M., \& França, J. R. A. (2016). Global, diffuse and direct solar radiation at the surface in the city of Rio de Janeiro: Observational characterization and empirical modeling. Renewable Energy, 91, 64-74. https://doi.org/10.1016/j.renene.2016.01.040
Parinacota, A., Cornejo, L., Martín-pomares, L., \& Alarcon, D. (2017). A through analysis of solar irradiation measurements in the region of. 112. https://doi.org/10.1016/j. renene.2017.04.012

Scarpa, F., Bianco, V., \& Tagliafico, L. A. (2018). A clear sky physical based solar radiation decomposition model. Thermal Science and Engineering Progress, 6, 323-329. https://doi.org/10.1016/j.tsep.2017.11.004

Sengupta, M., Xie, Y., Lopez, A., Habte, A., Maclaurin, G., \& Shelby, J. (2019). The National Solar Radiation Data Base ( NSRDB ). Renewable and Sustainable Energy Reviews, 89(March 2018), 51-60. https://doi.org/10.1016/j. rser.2018.03.003

Tahir, Z. R., \& Asim, M. (2018). Surface measured solar radiation data and solar energy resource assessment of Pakistan: A review. In Renewable and Sustainable Energy Reviews (Vol. 81, pp. 2839-2861). https://doi.org/10.1016/j. rser.2017.06.090

Zawilska, E., \& Brooks, M. J. (2011). An assessment of the solar resource for Durban, South Africa. Renewable Energy, 36(12), 3433-3438. https://doi.org/10.1016/j. renene.2011.05.023 